\title{
Digital darkfield tableaus
}

P. Fraundorf*, Jinfeng Wang*, Eric Mandell*, and Martin Rose* *Physics \& Astronomy and Center for Molecular Electronics, University of Missouri-St.Louis (63121), St.Louis, MO, USA

Like wavelet decompositions, power spectra "tiled with direct space maps" are versions of a lattice image intermediate between direct and reciprocal space [1]. They generally take about the same time to calculate as a power spectrum, and contain useful information of all sorts about "where periodicities lie". Questions of this sort will be of increasing importance e.g. in subångstrom resolution pictures. The information is useful in spite of the fact that members of the mathematical harmonic analysis community have long moved past the sharp edged Fourier apertures that electron optical darkfield requires [2]. By illustrating useful applications for sharp-edged tilings, we might thus inspire the math community to look at the much wider range of pattern recognition problems that today's high resolution electron microscopists face.

The first tiling we discuss is the one most familiar to microscopists: Amplitude tableaus. It's familiar because the inverse transform (bandpass image) of an apertured Fourier transform (or diffraction pattern) has phase and amplitude components, but only the latter are detectable in optical darkfield images recorded on film. They are also optically tedious to set up. Digitally on the other hand, a 1024x1024 image yields a tableau of 32x32 complex bandpass images in only seconds on a modern computer, the amplitude version of which corresponds (in terms of bandpass) to 1023 darkfield and one brightfield image taken with the microscope. Because individual tiles with spatial correlations easily catch one's eye, one can quickly examine the tableau for regions of hidden periodicity, as well as for regions of characteristic shape (like the bowties and butterflies characteristic of icosahedral twins in the example of Fig. 1).

The other tilings we mention here are Isotropic and Shear Strain tableaus. These exploit the idea that phase in the bandpass image measures displacement, so that phase gradient measures periodicity deviations from the bandpass reference [3]. This technique has been applied, one periodicity at a time, for example to measure interface and dislocation strain [4,5]. Fig. 2 illustrates how a tableau of isotropic (parallel to the reference g-vector) and shear (perpendicular to the g-vector) strain tiles in effect yield "diffraction contrast images" from a whole range of operating reflections. Here we use opposing hues red and cyan to represent relative compression and expansion, respectively, in the isotropic maps, and the orthogonal hues indigo and chartreuse to represent $\mathrm{CCW}$ and $\mathrm{CW}$ rotation, respectively, in the shear maps.

References:

[1] P. Fraundorf and G. K. Fraundorf, Proc. 47th Ann. EMSA Meeting (1989) 122-123

[2] P. Fraundorf, Digital Darkfield Decompositions (arXiv:cond-mat/0403017).

[3] O. Saxton, Synopsys (Synoptics, Cambridge, 1990) Spring-2.

[4] E. Snoeck et al, Thin Solid Films 319 (1998) 147-162.

[5] C. L. Johnson, M. J. Hÿtch and P. R. Buseck, Amer. Min. 89 (2004) 1374-1379. 

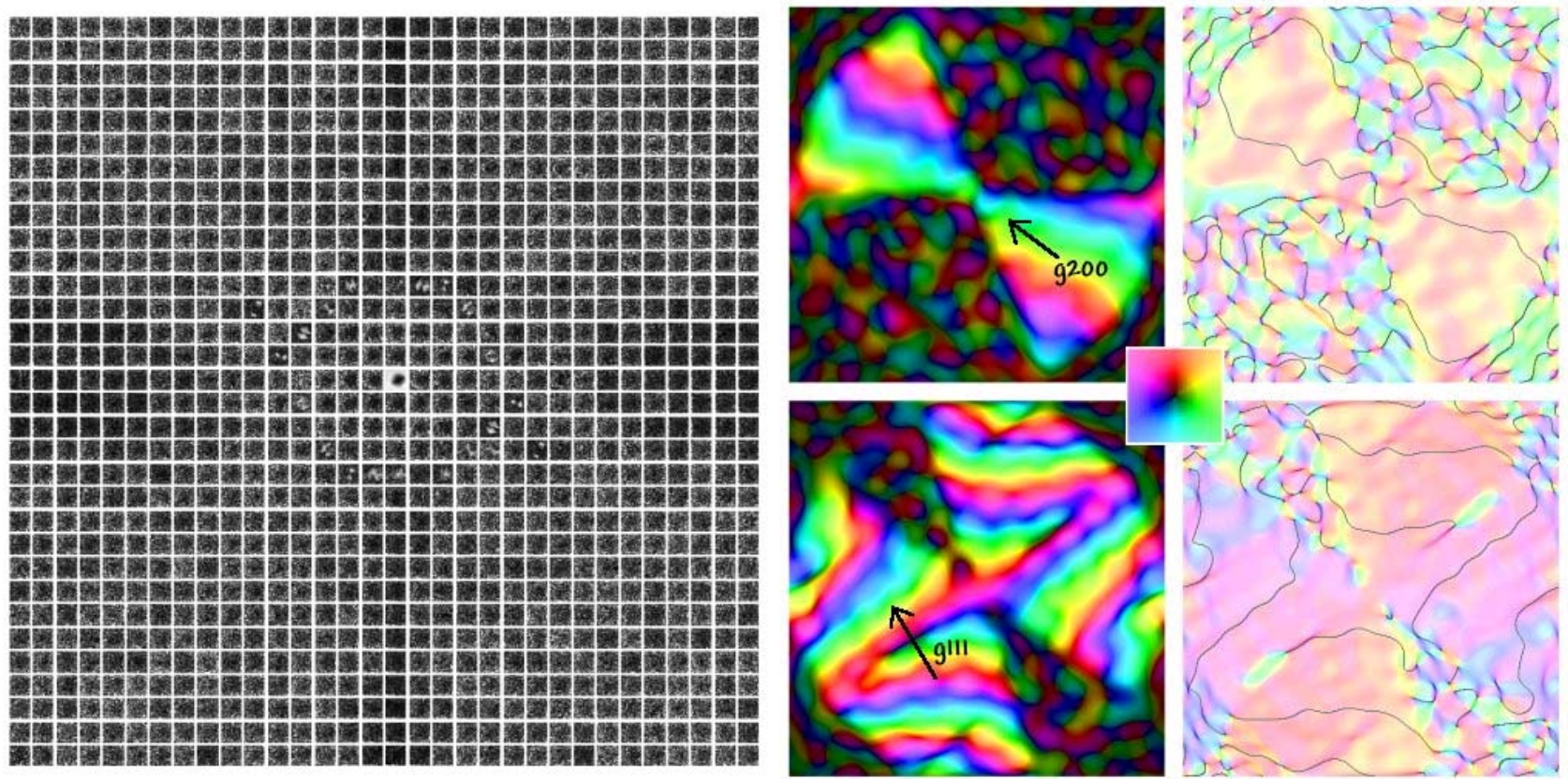

Fig. 1: At left is the $32 \times 32$ bandpass tableau of the 1024x1024 HREM image of an icosahedral Pd twin, viewed down one of it's 5-fold axes with an EM430ST at $300 \mathrm{kV}$. Tiny bowties and butterflies, like those at right, correspond to many diffraction spots around the brightfield image at center.

Applied to randomly-oriented ico-twins, the method also reveals these butterfly/bowtie signatures.
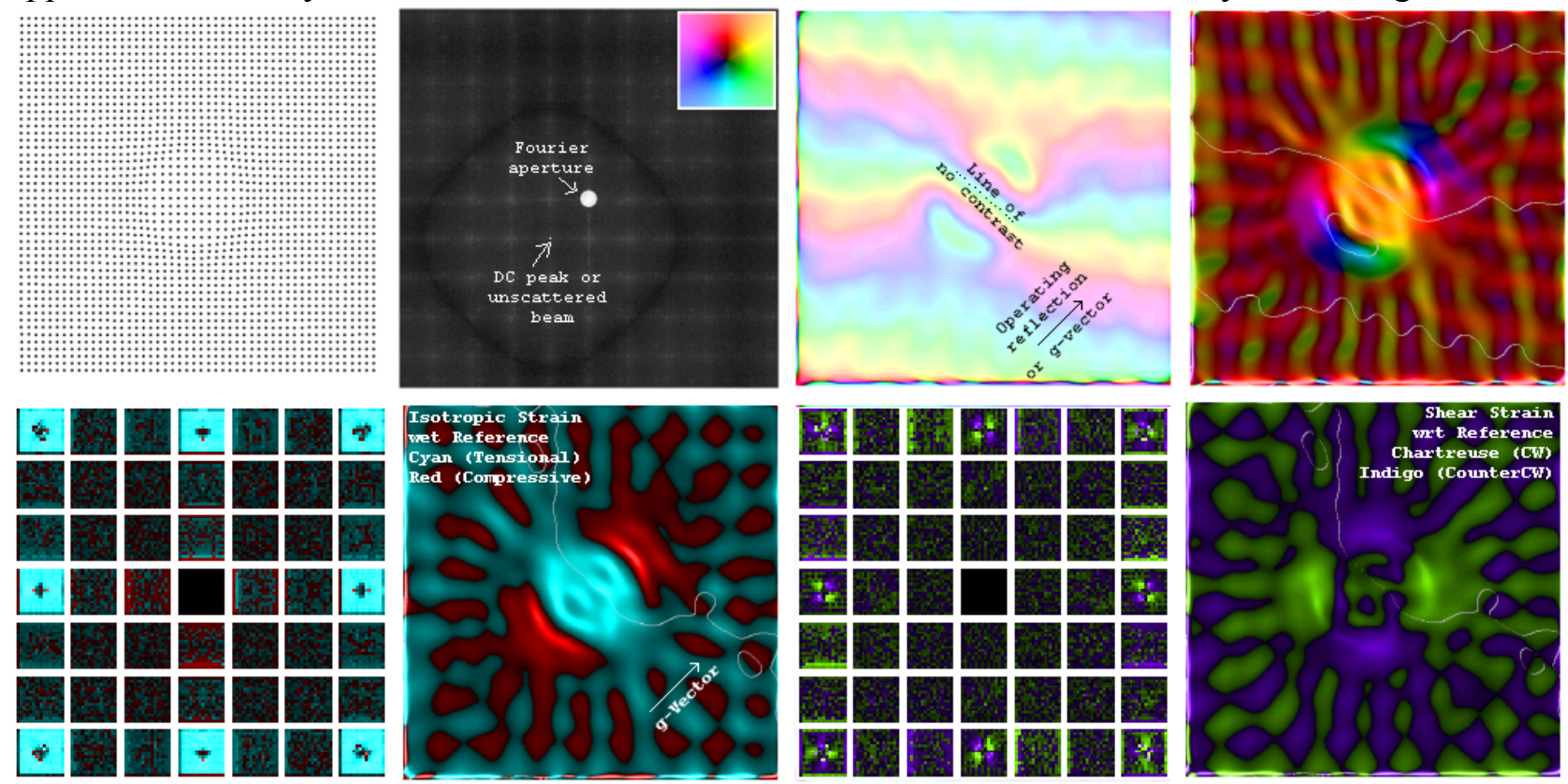

Fig. 2: The top row (from left to right) illustrates: projected potential of a simulated inclusion, offset diffraction pattern, complex color darkfield and component color strain. The bottow row excerpts the isotropic strain tableau, the isostrain map for one g-vector, the shear strain tableau, and the shear map for the same g-vector. Note that fringes rotated $\mathrm{CW}$ relative to $\mathrm{g}$ are charteuse, etc. 